\title{
Smart Plant Care Voice Recognition and Plant Disease Detection Using IOT
}

\author{
${ }^{1}$ Kulkarni Gauri N., ${ }^{2}$ Deshmukh Pratiksha K., ${ }^{3}$ Sawant Shital P., ${ }^{4}$ Kapadane Tejaswini R., \\ ${ }^{5}$ Prof. Pandav R.M.
}

\author{
1,2,3,4,5 Department of computer engineering, JES's Institute of Engineering, Babhulgaon, Nashik, \\ Maharashtra, India. 1'mail2kulkarnigauri@gmail.com, ${ }^{2}$ pratideshmukh4296@gmail.com, \\ ${ }^{3}$ shitalsawantm@gmail.com, ${ }^{4}$ tejaswinikapadne1999@gmail.com, ${ }^{5}$ roshan.pandav67@gmail.com
}

\begin{abstract}
Nowadays Internet of Things (IoT) and Remote Sensing (RS) techniques are used in different area of research for monitoring, collecting and analysis data from remote locations. Identification of the plant diseases is the key to preventing the losses in the yield and quantity of the agricultural product. The studies of the plant diseases mean the studies of visually observable patterns seen on the plant. Health monitoring and disease detection on plant is very critical for sustainable agriculture. It is very difficult to monitor the plant diseases manually. It requires tremendous amount of work, expertise in the plant diseases, and also require the excessive processing time. Hence, image processing is used for the detection of plant diseases and also care of plant using voice recognition. Through the Internet, Remote Data Center gets the real-time water quality data, and then analysis, process and record the dataGeneral Terms
\end{abstract}

Keywords: IOT, Sensor, Plant Disease, Image Processing.

\section{INTRODUCTION}

We In our proposed system we are implementing smart plant care using voice recognition and detecting plant disease image processing. With help of smart plant talk we can care plant time to time and also check disease on plant this application much of help to farmer or agriculture department. We are implementing care of plant using voice recognition we suggest suitable farming to farmer and also which disease occur due to impure water, Plant Disease Detection. In daily basis we found no of diseases and increase death ratio due to impure water and also not getting suitable farming also is day by day increasing. In our proposed system we overcome all this problem using Smart water quality measuring. Also suggest best farming to the farmer, our proposed system use for household and we are implementing water bacteria detection using image processing.

\section{SENSORS DETAILS}

\subsection{ArduinoKit (Uno)}

The Starter Kit walks you through the basics of using the Arduino in a hands-on way. You'll learn through building several creative projects. The kit includes a selection of the most common and useful electronic components.

\section{$2.2 \mathrm{pH}$ sensor}

$\mathrm{A} \mathrm{pH}$ meter is a scientific instrument that measures the hydrogen-ion activity in water-based solutions, indicating its acidity or alkalinity expressed as $\mathrm{pH}$.

\subsection{Moisture sensor}

Turbidity sensors measure the amount of light that is scattered by the suspended solids in water. As the amount of total suspended solids (TSS) in water increases, the water's turbidity level (and cloudiness or haziness) increases.

\subsection{Cables}

Electrical cables are used to connect two or more devices, enabling the transfer of electrical signals or power from one device to the other. Cables are used for a wide range of purposes, and each must be tailored for that

\section{PROPOSED SYSTEM}

In our proposed system we are helping to farmer and peoples to care their plants and farm using new innovative system of IOT and sensors. Our system help to improve life plants and more production of plants. Plants can tell us what is affecting them with the electrical signals they emit with the help of (Internet of things) IoT.In such ways that is your plant thriving under current light levels? Is your plant thirsty? Or even satisfy with your care? This IoT project describes how to build a project that monitors the plant health status by keeping contact with the owner 


\section{MODULES}

1. Training Module Here Expert can add disease infected images. Here GLCM algorithm can extract features from Image. And disease name.

2. Detection Module Here User or farmer can input sample images and if image feature is match with train dataset then plant disease will found or detected.

3.Plan care Detection in this module plant can communicate with human being so we can care of plant

\section{LITERATURE SURVEY}

[1] J. Santos, J. J. Rodrigues, J. Casal, K. Saleem, and V. Denisov, The Internet has emerged as a key network to make information accessible quickly and easily, revolutionizing how people communicate and interact with the world. The information available on the Internet about a given subject

may be extensive, allowing the development of new solutions to solve people's day-to-day problems. One such solution is the proposal of intelligent personal assistants (IPAs), which are software agents that can assist people in many of their daily activities. IPAs are capable of accessing information from databases to guide people through different tasks, deploying a learning mechanism to acquire new information on user performance. IPAs can improve the assistance they offer to users by collecting information autonomously from objects that are available in the surrounding environment. To make this idea feasible, IPAs could be integrated into ubiquitous computing environments in an Internet of Things (IoT) context. Therefore, it is necessary to integrate wireless sensor networks with the Internet properly, considering many different factors, such as the heterogeneity of objects and the diversity of communication protocols and enabling technologies. This approach fulfills the IoT vision. This paper surveys the current state of the art of IoT protocols, IPAs in general, and IPAs based on IoTs..

[2]A. Belouchrani, K. Abed-Meraim, J. F. Cardoso, and E. Moulines ;Mohsin RezaSeparation of sources consists of recovering a set of signals of which only instantaneous linear mixtures are observed. In many situations, no a priori information on the mixing matrix is available: The linear mixture should be "blindly" processed. This typically occurs in narrowband array processing applications when the array manifold is unknown or distorted. This paper introduces a new source separation technique exploiting the time coherence of the source signals. In contrast with other previously reported techniques, the proposed approach relies only on stationary second-order statistics that are based on a joint diagonalization of a set of covariance matrices. Asymptotic performance analysis of this method is carried out; some numerical simulations are provided to illustrate the effectiveness of the proposed method.
[3].Muslima, Umme, and M. Babul Islam. Deals with the recognition process of Bangla speech. The used database consists of two sets of data-one is for training containing 3824 utterances of Bangla digit sequences of 25 male and 25 female speakers and the other one is test dataset containing 1985 utterances of 26 male and 26 female speakers. The test set is subdivided into four groups such as clean1, clean2, clean3 and clean4. Mel-LPC based front-end has been used to design the front-end, since it incorporate auditory-like frequency resolution. The MelLPC is a time-domain feature and computationally efficient. The Mel-LPC based cepstral coefficients are obtained directly from the input speech by using generalized autocorrelation function. In this estimation process bilinear transformation is not required, and frequency warping is obtained by using a first-order allpass filter instead of unit delay. A detail experimental framework both for front-end and HMM based back-end have been presented in this paper. The final recognition experiments show the satisfactory performance of the developed system. The recognition accuracy are found to be $98.11 \%, 98.05 \%, 97.94 \%$, and $97.63 \%$, for test sets clean1, clean2, clean3 and clean4, respectively.

[4].Budiharto, Widodo, and DerwinSuhartono ;the objective of this paper is to propose an intelligent service robot in indoor environment with voice recognition and telepresence capabilities. We have assumed that this model of service robot is used to deliver a drinking cup to the recognized customer from the starting point to the destination. We have developed and introduced 3 main modules to avoid obstacle, to recognize commands and telepresence capabilities based on Google App Engine. The detection of obstacles, which is based on the information from the 3 ultrasonic sensors, uses an Interval type-2 Fuzzy Logic for adopting uncertainty. The main contribution of this research is to provide a complete mechanism of communication model between main controllers to other modules in service robot. We present the results of the experiment of the service robot called BeeBot III which implements our proposed method and we also evaluate its performance.

[5].Thiang, DhannyWijaya ;Automatic speech recognition by machine has been a goal of a research for a long time, which concurrent the inter desciplines like mechanical, electronics and coputer engineering. Speech recognition is the process of converting an acoustic signal, captured by a microphone or a telephone, to a set of words. The recognized words can be the final results, as for applications such as commands \& control, data entry, and document preparation. They can also serve as the input to further linguistic processing in order to achieve speech understanding. The speech recognition system has also been implemented on some particular devices. Some of them are personal computer (PC), digital signal processor, and another kind of single chip integrated circuit. In this 
paper we propose voice recognition to control robot using finger print comparison by Euclidean square distance, band pass filters and java technology

\section{SYSTEM ARCHITECTURE}

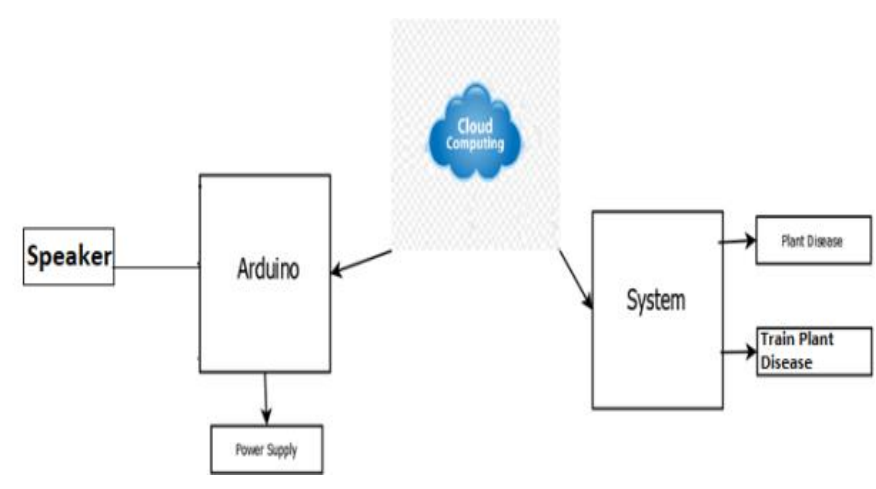

Fig 1. Architecture

In this architecture this architecture we are using arduinoUno.

In our system arduino board is connected to power supply.

And other sensors are connected to arduino sense all sensor data of plant and store in cloud on the basis of those parameter plant can be speak also we are help farmer find plant disease using image processing.

\subsection{Module:}

\subsubsection{Admin:}

First admin can login to system and train images with help of GLCM.Images is uploaded into the system. In GLCM we are extracting image features.

\subsubsection{View Train Data:}

In this module Admin can view train data.

\subsubsection{Smart Irrigation system:}

In automatic irrigation system we will measure soil parameters like soil moisture, $\mathrm{PH}$ Value, temperature of soil in the farm and according to the soil moisture it take decision to turn on/off the water pump through Ardunio and it will give notification on user mobile phone.

\subsubsection{Disease Detection}

In disease detection we used GLCM algorithm and will identify disease through image processing. Following are the steps of disease detection using image processing.

\subsubsection{Water Quality Indicator}

In water quality detection, we detect the water quality through turbidity sensor it will measure the water parameter like potential Hydrogen (PH), oxidation and reduction potential [ORP], temperature.

\section{Technique}

In this paper we applying different technique

\subsection{IOT}

The Internet of things is the extension of Internet connectivity into physical devices and everyday objects.
Embedded with electronics, Internet connectivity, and other forms of hardware, these devices can communicate and interact with others over the Internet, and they can be remotely monitored and controlled.

\subsection{Arduino}

Arduino is an open-source hardware and software company, project and user community that designs and manufactures single-board microcontrollers and microcontroller kits for building digital devices.

\subsection{Ph sensor}

A $\mathrm{pH}$ meter is a scientific instrument that measures the hydrogen-ion activity in water-based solutions, indicating its acidity or alkalinity expressed as $\mathrm{pH}$

\subsection{Turbidity sensors}

Measure the amount of light that is scattered by the suspended solids in water. Turbidity sensors are used in river and stream gaging, wastewater and effluent measurements, control instrumentation for settling ponds, sediment transport research, and laboratory measurements.

\subsection{Image Processing}

Image processing is the use of computer algorithms to perform image processing on digital images. As a subcategory or field of digital signal processing, digital image processing has many advantages over analog image processing.

\subsection{GLCM}

The GLCM is a tabulation of how often different combinations of pixel brightness values (grey levels) occur in an image.

GLCM texture considers the relation between two pixels at a time, called the reference and the neighbour pixel. In the illustration below, the neighbour pixel is chosen to be the one to the east (right) of each reference pixel

\subsection{Feature Extraction}

Various features are extracted using feature extraction techniques. This precisely describes the diseased region based on color, shape and textural features. Various feature extraction method such as colour, co-occurrence, skewness, contrast, correlation etc are used to extract the desired set of features.

\section{ALGORITHM}

Begin

Step 1 call the algorithm computing GLCM matrix in four direction $(0,45,90,135)$ with distance $\mathrm{d}=1$

Step 2 Call the algorithm of normalizing each GLCM matrix

Step 3 for each GLCM matrix in certain angle

I. Calculate Image Features according to their equations. 
II. Store computed features in vector.

End.

\section{Result}

In Our System Result will be

\section{Train System}

Here System will be Train means here expert can add plant disease infected Images to system means system can build Dataset of Diseases infected images.

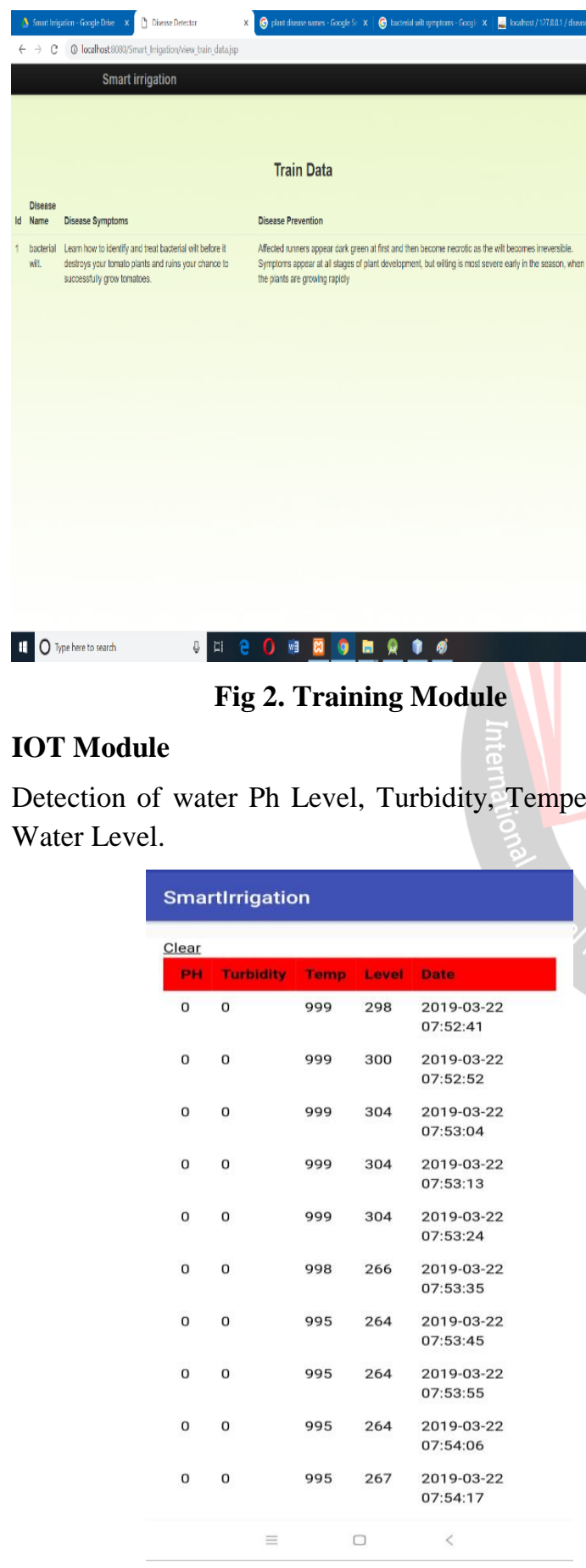

Fig 3.Water Parameter

\section{Disease Detection}

In this module plant disease is detected.

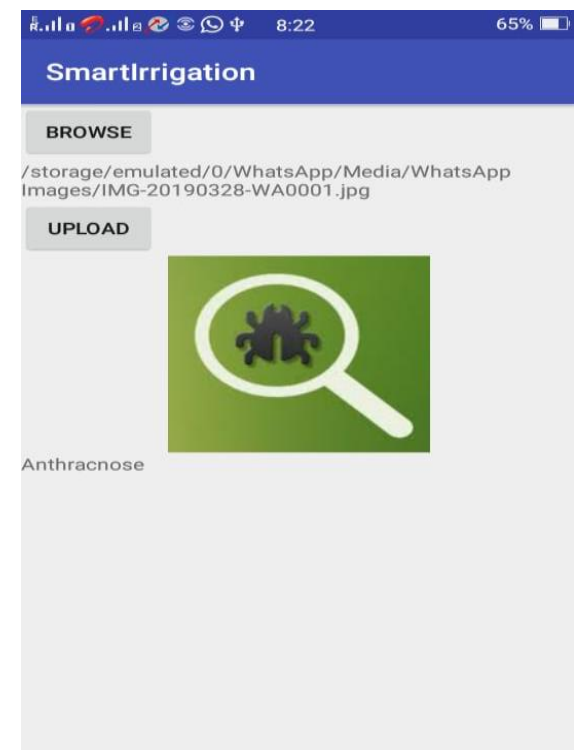

Fig 4.Disease Detection

Analysis

\section{GLCM Algorithm}

\section{Analysis}

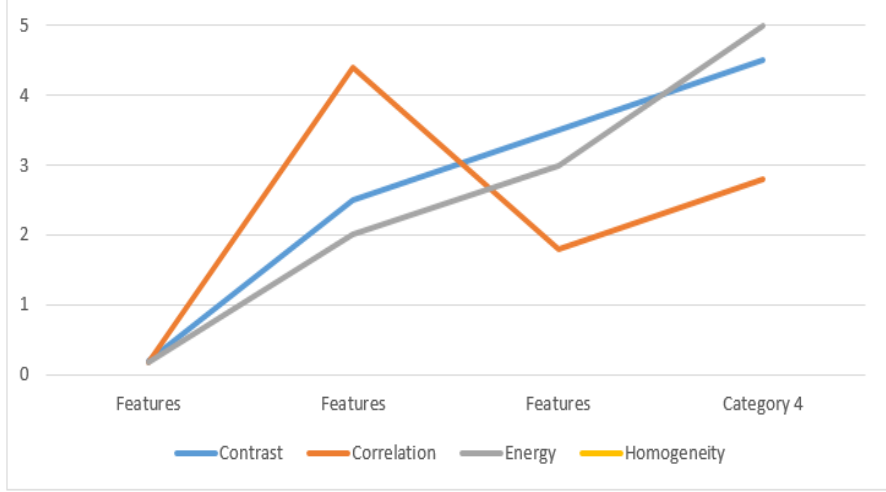

Fig 5. Analysis

Features of GLCM explain below

\section{Angular Second Moment}

Angular Second Moment is also known as Uniformity or Energy. It is the sum of squares of entries in the GLCM Angular Second Moment measures the image homogeneity. Angular Second Moment is high when image has very good homogeneity or when pixels are very similar

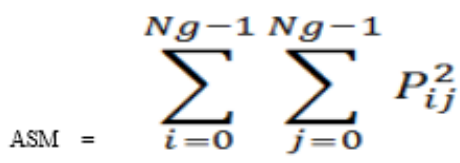

\section{Equation 1}

Where $i, j$ are the spatial coordinates of the function $p(i$, $\mathrm{j}), \mathrm{Ng}$ is gray tone. 


\section{Inverse Difference Moment}

Inverse Difference Moment (IDM) is the local homogeneity. It is high when local gray level is uniform and inverse GLCM is high.

$$
\mathrm{DM}=\frac{\sum_{i=0}^{N g-1} \sum_{j=0}^{N g-1} P_{i j}}{1+(i-j)^{2}}
$$

IDM weight value is the inverse of the Contrast weight.

\section{Entropy}

Entropy shows the amount of information of the image that is needed for the image compression. Entropy measures the loss of information or message in a transmitted signal and also measures the image information.

$$
\sum_{\mathrm{ENTROPY}}=\sum_{i=0}^{N g-1}-P_{i j} * \log P_{i j}
$$

\section{Correlation}

Correlation measures the linear dependency of grey levels of neighboring pixels. Digital Image Correlation is an optical method that employs tracking \& image registration techniques for accurate $2 \mathrm{D}$ and $3 \mathrm{D}$ measurements of changes in images. This is often used to measure deformation, displacement, strain and optical flow, but it is widely applied in many areas of science and engineering. One very common application is for measuring the motion of an optical mouse.

\section{CONCLUSION}

The main aim of this project work is develop a real time plant care and detection of plant diseases using image processing. This system help to farmer care plant using voice reorganization, in this system plant can speack what it need with help of senosors.this is most innovative for plant care.

\section{REFERENCES}

[1] K.Lokesh Krishna, Omayo Silver, Wasswa Fahad Malende, K.Anuradha, "Internet of Things application for Implementation of Smart Agriculture System", International conference on I-SMAC (IoT in Social, Mobile, Analytics and Cloud) (I-SMAC 2017).

[2] K.Lokesh Krishna, Omayo Silver, Wasswa Fahad Malende, K.Anuradha, "Internet of Things application for Implementation of Smart Agriculture System", International conference on I-SMAC (IoT in Social, Mobile, Analytics and Cloud) (I-SMAC 2017).

[3] J. Santos, J. J. Rodrigues, J. Casal, K. Saleem, and V. Denisov, "Intelligent personal assistants based on internet of things approaches," IEEE Systems Journal, 2016.
[4] Budiharto, Widodo, and DerwinSuhartono. "Intelligent service robot with voice recognition and telepresence capabilities." In SAI Intelligent Systems Conference (IntelliSys), 2015, pp. 301-304. IEEE, 2015.

[5] J. Gubbi, R. Buyya, S. Marusic, and M. Palaniswami, "Internet of Things (IoT): A vision, architectural elements, and future directions," Future Generation Computer Systems, vol. 29, no. 7, pp. 1645-1660, Sep. 2013.

[6] "Cloud Speech-to-Text - Speech Recognition," Google Cloud. [Online]. Available: https://cloud.google.com/speech-to-text/.

[7] “Alexa Voice Service." [Online]. Available: https://developer.amazon.com/alexa-voice-service.

[8] "Introducing Wit Speech API." [Online]. Available: https://wit.ai/blog/2014/02/12/speech-api.

[9] Muslima, Umme, and M. Babul Islam. "Experimental framework for mel-scaled LP based Bangla speech recognition." Computer and Information Technology (ICCIT), 2013 16th International Conference on. IEEE, 2014.

[10] Budiharto, Widodo, and DerwinSuhartono. "Intelligent service robot with voice recognition and telepresence capabilities." In SAI Intelligent Systems Conference (IntelliSys), 2015, pp. 301-304. IEEE, 2015.

[11] Haro, Luis Fernando D., et al. "Low-cost speaker and language recognition systems running on a Raspberry Pi." IEEE Latin America Transactions 12.4 (2014): 755-763.

[12] Thiang, DhannyWijaya. "Limited speech recognition for controlling movement of mobile robot implemented on atmega162 microcontroller." In Computer and Automation Engineering, 2009. ICCAE'09. International Conference on, pp. 347-350. IEEE, 2009.

[13] Fezari, Mohamed. "New speech processor and ultrasonic sensors based embedded system to improve the control of a motorised wheelchair." Journal of Applied Sciences Research5.10 (2009): 17501755.

\section{About Authors}

Miss.Kukarni Gauri N. ${ }^{1}$, She perusing in BE from S.N.D. College of Engineering \& RC, Yeola. His main area of interest is Smart Plant Care Voice Recognition and Plant Disease

Detection Using IOT.

Miss.Sawant Shital $\mathbf{P}^{2}$, She perusing in BE from S.N.D. College of Engineering \& RC,Yeola. His main area of interest is Smart Plant Care Voice Recognition and Plant Disease Detection Using IOT.

Miss.Kapadne Tejaswini R. ${ }^{3}$, She perusing in BE from S.N.D. College of Engineering \& RC, Yeola. His main area of interest is Smart Plant Care Voice Recognition and Plant Disease Detection Using IOT.

Miss.Deshmukh Pratiksha K. ${ }^{\mathbf{4}}$, She perusing in BE from S.N.D. College of Engineering \& RC,Yeola. His main area of interest is Smart Plant Care Voice Recognition and Plant Disease Detection Using IOT. 